\title{
Notable efecto hipotensor de los cambios del estilo de vida. Ensayo clínico PREMIER
}

Effects of Comprehensive Lifestyle Modification on Blood Pressure Control. Main Results of the PREMIER Clinical Trial. Appel, L. J. y cols. JAMA 2003 April 23/30; 289:2083-2093.

\section{Objetivo}

Determinar el efecto de cambios en el estilo de vida sobre la presión ar terial mediante la implementación simultánea de 3 modelos diferentes de educación.

Diseño

Ensayo clínico aleatorizado y controlado.

\section{Lugar}

Realizado en 4 centros clínicos de EE.UU.

\section{Pacientes}

Se incluyeron 810 adultos sanos sin fármacos antihipertensivos con registros de presión arterial (PA) entre 120-159 mmHg de PA sistólica (PAS) y 80-95 mmHg de PA diastólica (PAD).

\section{Intervención}

Asignación aleatoria a tres grupos: 1) grupo con modificación de estilo de vida por intervención conductual denominado "establecido" (implementación de las recomendaciones de cambio del estilo de vida establecidas: pérdida de peso, reducción de ingesta de sodio, aumento de actividad física y reducción de ingesta de alcohol; $n=268$, "grupo E"), 2) grupo "establecido con la implementación de la dieta DASH" ( $n=269$, "grupo ED"), y 3) grupo "solo consejo" ( $n=273)$.

Los tres grupos fueron semejantes en edad y PA. Se realizó evaluación de resultados a los seis meses.

En el grupo "sólo consejo" un dietista discutió individualmente en 30 minutos los factores de riesgo que afectan la PA y entregó material escrito.No hubo más contacto hasta los seis meses. Los otros dos grupos (E y ED) recibieron 18 sesiones en seis meses con una intervención orientada al comportamiento.

\section{Medición de resultados principales}

Los objetivos de los grupos E y ED fueron: una pérdida de peso de al menos $6,8 \mathrm{~kg}$ (en aquéllos con sobrepeso-obesidad), activi- dad física (intensidad moderada) > 180 minutos por semana, reducción de la ingesta calórica, un consumo diario de sodio de no más de $100 \mathrm{mEq}$ y restricción del consumo de alcohol, y en el grupo ED, consumo de vegetales y frutas (9-12 porciones/día), productos lácteos descremados (2-3 porciones/día).

\section{Resultados principales}

En los grupos E y ED hubo una reducción significativa del peso, aumentaron significativamente el entrenamiento y redujeron la ingesta de sodio. En el grupo ED también aumentó significativamente la ingesta de frutas, vegetales, lácteos, calcio y potasio en comparación con los otros grupos. Un tercio del grupo ED, y sólo un $6 \%$ de los otros dos grupos alcanzaron el objetivo de consumo de frutas y vegetales.

La reducción promedio de la PA (y entre paréntesis reducción en los hipertensos) fue:PAS de $6,6(7,8) \mathrm{mmHg}$ en el "sólo consejo", $10,5(12,5) \mathrm{mmHg}$ en el $\mathrm{E}, \mathrm{y} 11,1(14,2) \mathrm{mmHg}$ en el ED.Las reducciones de la PAD fueron de $3,8(3,8) \mathrm{mmHg}, 5,5(5,8) \mathrm{mmHg}$, y $6,4(7,4) \mathrm{mmHg}$, respectivamente.

La prevalencia de hipertensión al inicio del estudio estaba entre el 37 y $38 \%$ en los distintos grupos, mientras que al final era del $26 \%$ en el grupo consejo, $17 \%$ en el $E$ ( $p=0,01$ comparado con el primero), y del $12 \%$ en el ED ( $p<0,001$ comparado con el grupo consejo), sin diferencias significativas al comparar con el grupo establecido $(p=0,12)$.

La prevalencia de PA óptima fue del $19 \%$ en el grupo consejo, del $30 \%$ en el $E(p=0,005)$ y del $35 \%$ en el ED $(p<0,001 ; y p=0,24$ en comparación con el grupo E).

\section{Conclusiones}

Los resultados de este estudio demuestran que el cambio de estilo de vida a través de intervenciones conductuales es factible, reduce la $\mathrm{PA}$, controla la hipertensión arterial y reduce la prevalencia de hipertensión en pacientes de mediana edad.Los beneficios se extienden a pacientes en riesgo de tener hipertensión arterial.

Fuente de financiamiento: no referida.

\section{Comentario}

Se pueden resaltar aspectos importantes del presente estudio:

1) El tratamiento no farmacológico puede ser muy efectivo cuando se implementa adecuadamente.

Confirma los resultados del estudio TONE (primer estudio clínico multicéntrico que demostró el efecto beneficioso de las modificaciones del estilo de vida en el control de la hipertensión arterial), en el que a los 30 meses el $30 \%$ de los pacientes logró no requerir más medicación y los pacientes bajo dieta hiposódica y que perdieron peso requirieron la mitad de la medicación hipotensora ${ }^{1-2}$.

2) Las reducciones de la PA registradas en este estudio son semejantes a las observadas en los ensayos clínicos con fármacos, y sabemos que los efectos beneficiosos son de gran impacto;por ejemplo, una pequeña reducción de la PA (5-6 mmHg en la PAD) en pocos años (3-6 años) reduce el riesgo cardiovascular en un $42 \%$ para accidente cerebrovascular (ACV) y un $14 \%$ para enfermedad coro- naria $^{3}$ y el descenso de PAS de $11 \mathrm{mmHg}$ está asociado a $36 \%$ de reducción de ACV, $27 \%$ de reducción de IAM no fatal y muerte por causa coronaria 4 . son sumamente efectivas, pero tengamos en cuenta que en los grupos $\mathrm{E}$ y ED la cantidad de horas destinadas a los encuentros educativos no es lo habitual en la práctica diaria.

Conclusión del comentador: Esta es la primera experiencia de dieta DASH en pacientes que manejan su alimentación.

En base a los resultados de este y otros estudios, se confirma la necesidad de modificar las estrategias de implementación del tratamiento no farmacológico de la hipertensión arterial, basado función de sodio, pérdida de peso y aumento de la actividad física.
3) El efecto de las inter venciones sobre la conducta y la educación damentalmente en la modificación del hábito alimentario y la restric-

Dr. Marcelo A. Rada [ Sección Hipertensión.Servicio Clínica medica, Hospital Italiano de Buenos Aires ]

Referencias

1) Sodium Reduction and Weight Loss in the Treatment of Hypertension in Older Persons: A Randomized Controlled Trial of Nonpharmacologic Interventions in the Elderly (TONE) Whelton PK.;Appel LJ.; Cutler JA.Y col.JAMA. 1998;279:839-846.

2) Effects of Reduced Sodium Intake on Hypertension Control in Older Individuals:Results From the Trial of Nonpharmacologic Interventions in the Elderly (TONE)

Appel LJ.; Espeland MA, Lacy CR.Arch Intern Med. 2001;161:685-693.

3) Blood pressure, stroke, and coronary heart disease.Part 2, Short-term reductions in blood pressure:overview of randomised drug trials in their epidemiological context.Lancet.1990.7;335:827-38. 4) Shep Cooperative Reserch Group. Prevention of stroke by antihypertensive drug treatment in older persons with Isolated Systolic hypertension. JAMA 1991;265:3255-3264. 